\title{
Effects of Nozzle and Contact Geometry on Arc Voltage in Gas Circuit-Breakers
}

\section{Conference Paper}

Author(s):

Bort, Lorenz (iD; Franck, Christian (1)

Publication date:

2016

Permanent link:

https://doi.org/10.3929/ethz-b-000123381

Rights / license:

In Copyright - Non-Commercial Use Permitted

Originally published in:

https://doi.org/10.1109//CHVE.2016.7800700 


\title{
Effects of Nozzle and Contact Geometry on Arc Voltage in Gas Circuit-Breakers
}

\author{
Lorenz S.J. Bort, Christian M. Franck \\ High Voltage Laboratory (HVL) \\ ETH Zurich \\ Zurich, Switzerland \\ lbort@ethz.ch
}

\begin{abstract}
At the high voltage laboratory at ETH Zurich switching ares are investigated in order to understand the physical processes that determine the relationship between current and arc voltage in gas blast circuit breakers. Experiments are performed using a breaker prototype with many independently controllable parameters, and very versatile pulsed DC current source. Previous work showed that the gas pressure has a strong influence on the arc voltage, therefore changing the fluid dynamic conditions in which the arc burns can be used to create different $\mathrm{dU} / \mathrm{dI}$ characteristics. In the presented paper a method to quantify these changes is presented, and the impact of the axial position of the contacts in a model gas circuit breaker on the voltage as function of current are discussed. The influence of the contact position on the average $\mathrm{dU} / \mathrm{dI}$ curve were small, but the fluctuations around this average value change depending on the nozzle section in which the arc burns. These results will be used to improve the theoretical understanding of the different arc cooling mechanisms, which in turn should enable the design of new geometries that result in a more favorable arc voltage characteristics for passive oscillation HVDC circuit breaker topologies in the future.
\end{abstract}

Keywords- HVDC switchgear, Gas circuit breaker, MRTB, Arc voltage, HVDC circuit breaker)

\section{INTRODUCTION}

HVDC circuit-breakers which use a resonance principle for current interruption employ gas circuit-breakers in the main current path, and an LC-circuit connected in parallel which excites an oscillating current in the breaker path without using active components. This passive oscillation principle relies on a specific arc characteristic. If the $\mathrm{dU} / \mathrm{dI}$ characteristic of the switching arc is negative, i.e. higher current leads to a lower arc voltage, the oscillation between the LC path and the main path will increase in amplitude and eventually lead to a current zero in the main breaker. Today, this principle is widely used in metal return transfer breakers (MRTB). This concept could also be used in HVDC circuit breakers, if it is possible to decrease the time until current zero. This requires an arc characteristic $\mathrm{dU} / \mathrm{dI}<0$ at higher currents, to ensure breaking of fault currents. Additionally, the steeper the gradient of $\mathrm{dU} / \mathrm{dI}$, the faster the current zero creation due to stronger amplification of the oscillation.
Prior research by M. Walter at ETH Zurich showed that $\mathrm{dU} / \mathrm{dI}$ is negative only up to a certain current for a specific breaker geometry, but variables like nozzle geometry and blow pressure are powerful parameters that influence this arc characteristics. In particular, higher blow pressures shift the point where $\mathrm{dU} / \mathrm{dI}$ reaches zero (i.e. the voltage is constant independent of current) towards higher currents [1].

After the empirical measurements performed by Walter the research focus was shifted towards more theoretical work and the underlying physics, fluid dynamics in particular. Very detailed work on axially blown arcs was done already in the 1970s by Tuma, Lowke, Niemeyer, Hermann, Schade, Kogelschatz, Ragaller and other, which included measurements as well as simple 1D fluid dynamic calculations [2][3][4][5][6]. More recent black box models also exist, e.g. [7][8], as well as computational fluid dynamic simulations like [9] or [10]. Since most of this work was mainly focused on the behavior of AC circuit breakers near current zero, the voltage current relationship in the high current phase was not as important as it is for the DC breakers. Since measurement equipment and evaluation tools are more powerful today, this is a good starting point for deepening the physical understanding of how the voltage changes with current in DC arcs, and to find ways to influence voltage current characteristic in favor of

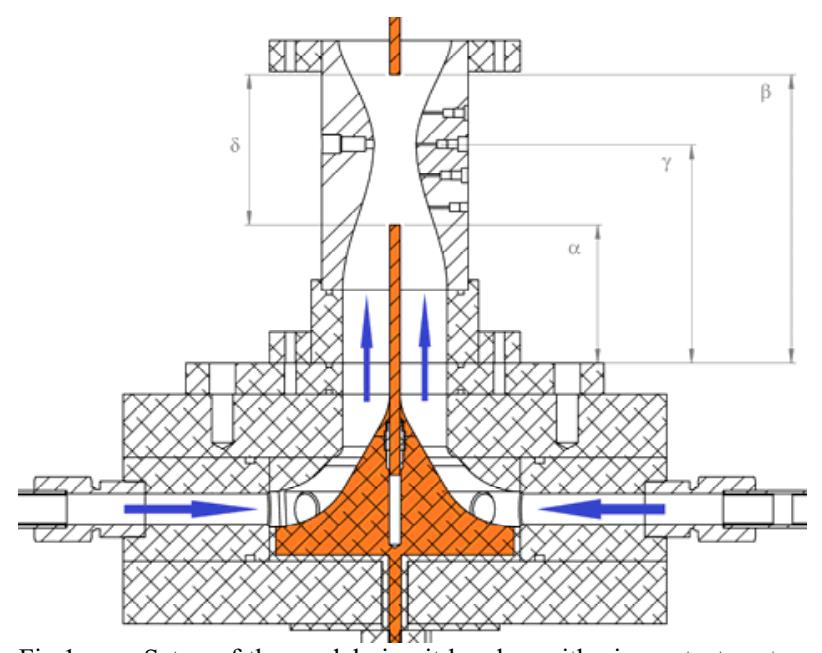

Fig 1. Setup of the model circuit breaker with pin contact system (orange), and single flow axial blowing (blue arrows). The inlet section and nozzle geometry has been optimized to create laminar flow conditions throughout the nozzle assembly. The contact pin position and the nozzle can be easily changed. The position of the upstream contact is indicated by $\alpha$, downstream contact position is $\beta$, nozzle throat position is at $\gamma$, and arc length is $\delta$. 
passive oscillation breaker topologies.

\section{EXPERIMENTAL SETUP}

\section{A. Model circuit breaker}

To study arcs, the setup described in [1] is used. The model circuit breaker includes independent contact movement and blowing systems, and uses air as blowing gas. In order to be able to use simple 1D fluid dynamic simulations for experiments without current (cold flow), the breaker model was adapted since the work of Walter (fig. 1 and 2). Sharp changes in cross section along the nozzle axis lead to flow separation from the wall and turbulent zones right before or after the edges. In order to obtain laminar flow conditions throughout the nozzle, the inlet section where the gas flow of the eight gas bottles of the blowing system is combined was redesigned, and the cylindrical nozzle was replaced by a converging-diverging design without any sharp changes in area (see fig. 1). At the same time, the upstream contact was replaced by a thinner pin with $5 \mathrm{~mm}$ diameter, which is closer to the effective arc diameter. The mounting of both contact pins allows for the axial position ( $\alpha$ and $\beta$ in Figure 1) to be varied. Therefore, experiments where the arc burns in different sections of the converging-diverging nozzle are possible. Using pins of only $5 \mathrm{~mm}$ in diameter is not feasible in real breakers since they would erode too fast. Nevertheless, thin pins were chosen because the previously used contact with $19 \mathrm{~mm}$ also represents a jump in fluid dynamic cross section at its end, which probably induces flow separation and 3D turbulences like Kármán vortices that cannot be accounted for in a $1 \mathrm{D}$ model. Additionally, the area of the contact should be much smaller than the area of the nozzle throat for the measurements described below, as changing the contact positions should influence flow conditions of the blowing gas as little as possible. With a minimal nozzle throat diameter of $20 \mathrm{~mm}$ and a contact pin of $5 \mathrm{~mm}$, the throat area decreases by only $6 \%$ if the upstream pin is moved downstream to (or past) the throat position which is considered negligible.

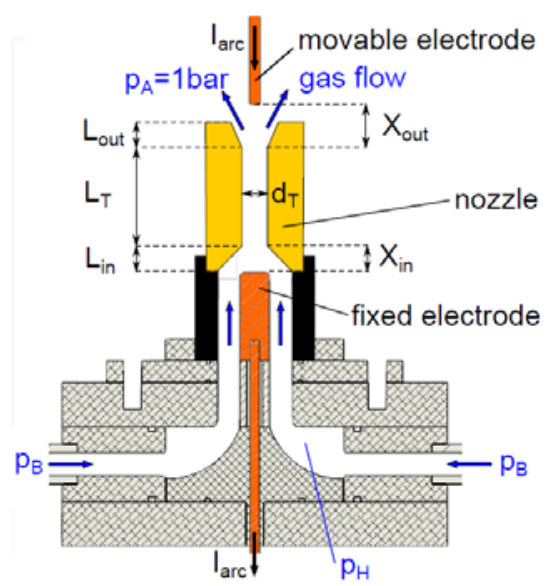

Fig 2. Previous setup as presented by Walter [1]. The nozzle was cylindrical and therefore has no defined throat, inlet and outlet are conical with sharp transitions in cross section. The upstream electrode is wide and blocks a significant part of the inlet area.

\section{B. Measurement Equipment}

To measure the $\mathrm{dU} / \mathrm{dI}$ of the arc precisely, measurement equipment with high accuracy and temporal resolution is needed. The voltage is measured using two commercial high bandwidth RC dividers connected to both contact pins of the breaker. Although the upstream electrode is connected to the same ground potential as the reference of the oscilloscopes, hard switching of power semiconductors in the current source introduce fast transients which are seen as common mode voltages due to the stray inductance of the connecting cables [11]. These are eliminated in post-processing by subtraction of the two voltage signals. The current is measured by a shunt resistor or a custom built current sensor as described in [11]. Voltage and current signals are recorded with a $12 \mathrm{bit}$ oscilloscope at a sampling rate of $100 \mathrm{MHz}$. This rate is faster than changes in arc voltage would require, but is needed for reliable common mode voltage rejection. Additionally, bandwidth can be traded for increased vertical resolution in post-processing by low pass filtering the signal, which was done using a Butterworth filter with cutoff frequency of $10 \mathrm{Mhz}$.

\section{METHOD}

\section{A. Determination of steady state dU/dI characteristics.}

To determine the voltage current characteristic of one particular nozzle and contact setup, the following sequence is run: a small pre-current below $200 \mathrm{~A}$ is created for about $70 \mathrm{~ms}$, during which the contacts separate and create an arc. When the movable contact reaches its final position, the valves of the blowing system are opened, and after the gas flow has stabilized, the current is ramped up to $1600 \mathrm{~A}$ and back to zero with a gradient of approximately $0.2 \mathrm{kA} / \mathrm{ms}$. For arc characterization, only this last high current phase is evaluated, where the contact positions and the blow pressure are constant. Figure 3 and figure 4 show the dU/dI curve of two single experiments. A general trend is visible, but the arc voltage can vary strongly at certain times, which makes it difficult to attribute one voltage value to a given current value. To compare different experimental setups, a metric was introduced that is capable of describing the interesting features of those curves. The voltage and current data is split up into bins, and for each bin the mean of the current, the median of the voltage, as well as the 5-percentile and the 95-percentile is determined. In order to gain more statistical significance, several experiments with identical conditions are combined. The result consists of two values for each current, which give the lower and upper limit of the arc voltage as a function of current. This way, not only a typical voltage current curve is obtained, but also the deviation from that mean voltage curve. In the work of Walter, the dU/dI curves were fitted with a simple two parameter model

$$
\mathrm{P}(\mathrm{g})=\mathrm{a} \cdot \mathrm{g}^{\mathrm{b}}
$$

In this equation, steady state is assumed, $\mathrm{P}$ is the arc power, $\mathrm{g}$ is its conductance, and $\mathrm{a}$ and $\mathrm{b}$ are fit parameters, with $\mathrm{b}$ usually close to 0.5 . For the changed setup, this equation was only able to fit the falling part of the dU/dI curves until 


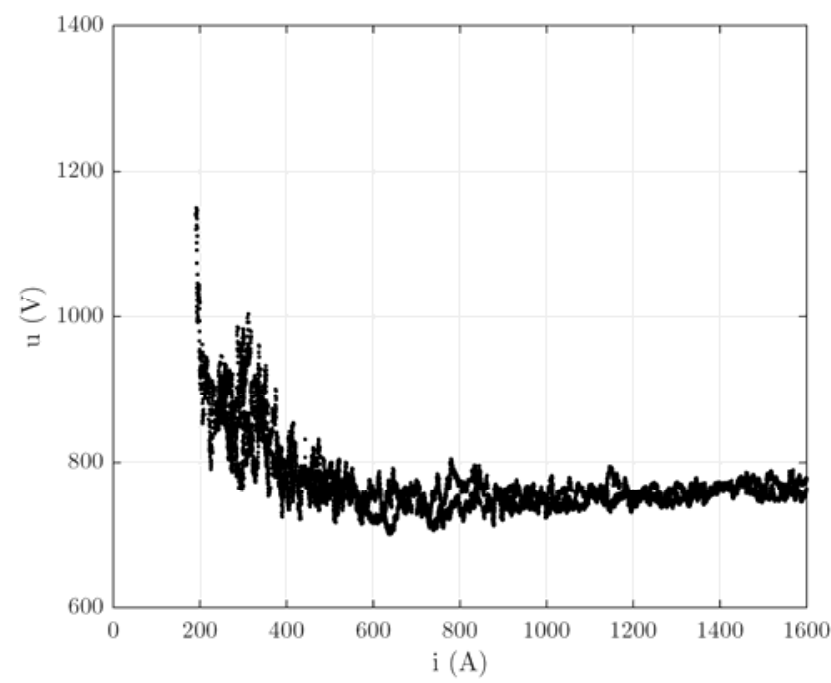

Fig. 3. Arc voltage as function of current. Arc length $\delta=80 \mathrm{~mm}$, upstream contact position $\alpha=75 \mathrm{~mm}$, downstream position $\beta=155 \mathrm{~mm}$. Shows one of the shots of series $5 \mathrm{~d}$ )

roughly 600 to $800 \mathrm{~A}$, and was therefore omitted in this work. In the future, an improved model, which is able to fit the results is highly desired. Such a model has to be able to describe different regimes, like the work of [7],[8] does by combining a Mayr and a Cassie term.

\section{RESULTS}

During several measurement series the axial position of the upstream and downstream contacts were systematically varied. The purpose of this variation was to determine which axial section of the arc contributes how much to the total arc voltage, and if the $\mathrm{dU} / \mathrm{dI}$ behaves differently when the arc is surrounded by subsonic or supersonic flow. In figure 5, one set of these measurements is presented. An arc of length $\delta=80 \mathrm{~mm}$ was moved downstream in $20 \mathrm{~mm}$ steps, each curve corresponds to six experiments, which were combined as described above. The same nozzle was used throughout the measurement sequence, its throat was at $\gamma=105 \mathrm{~mm}$ for all
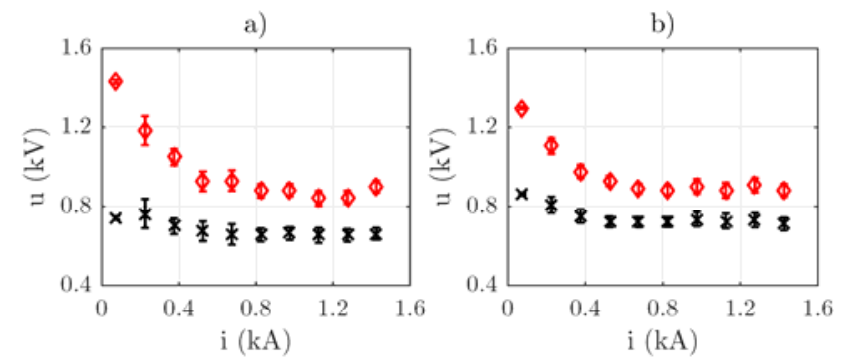

c)
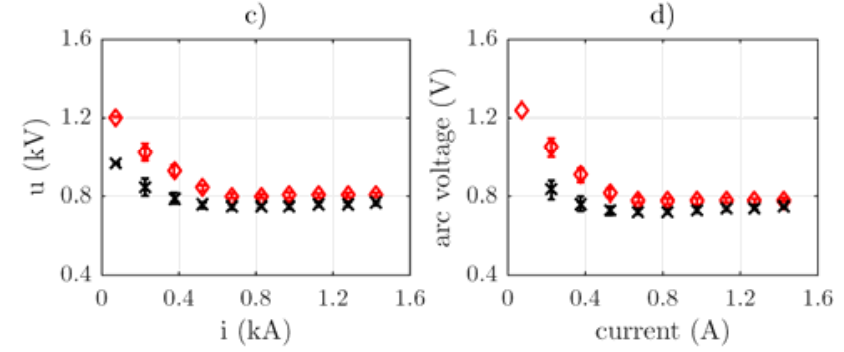

Fig. 5. Arc voltages for different axial contact positions, with constant arc length $\delta=80 \mathrm{~mm}$. a) $\alpha=15 \mathrm{~mm}, \quad \beta=95 \mathrm{~mm}, \quad$ ) $\alpha=35 \mathrm{~mm}, \beta=115 \mathrm{~mm}$, c) $\alpha=55 \mathrm{~mm}, \beta=135 \mathrm{~mm}$, d) $\alpha=75 \mathrm{~mm}$, $\beta=155 \mathrm{~mm}$. The black crosses indicate the 5 -percentile, i.e. minimum voltage, red diamonds show the 95 -percentile, i.e. maximum voltage, measured. Error bars mark the standard deviation of these values obtained by six shots in one experiment series with identical conditions.

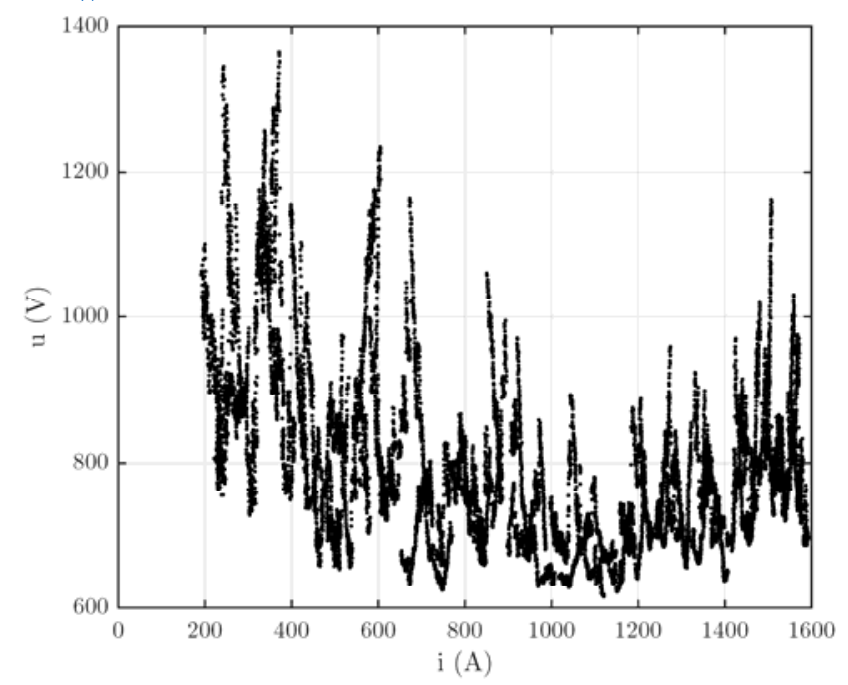

Fig. 4: Arc voltage as function of current. Arc length $\delta=80 \mathrm{~mm}$, upstream contact position $\alpha=15 \mathrm{~mm}$ from inlet, downstream position $\beta$ $=95 \mathrm{~mm}$. Shows one of the shots of series $5 \mathrm{a}$ )

presented measurements. Air was used as a blowing gas, the absolute pressure at the nozzle inlet was $7.5 \pm 0.5$ bar, exit pressure was 1 bar absolute.

Several changes can be observed in the results. Firstly, the minimum arc voltage is very similar in all cases. The differential arc resistance $\mathrm{dU} / \mathrm{dI}$ is negative for small currents and the voltage stays constant in the range between $700 \mathrm{~V}$ and $800 \mathrm{~V}$ for currents above 500A. For lower currents, the minimum voltage is higher, and $\mathrm{dU} / \mathrm{dI}$ of the black crosses is more negative for the cases c) and d) where the arc burns in the downstream part. Secondly, the maximum arc voltage follows the same trend. However, its value is significantly higher than the minimum in cases 5a) and 5b) where the arc mostly burns in the converging section of the nozzle. This means that there are voltage fluctuations of around $200 \mathrm{~V}$, as can be seen in figure 4 . The difference of minimum to maximum voltage is even higher at low currents below 500A. If a larger section of the arc is in the diverging nozzle section (5c and 5d) the maximum voltage approaches the minimum closely, which means there are almost no voltage fluctuations.

\section{DISCUSSION}

All the measurements show a similar overall trend. The differential arc resistance $\mathrm{dU} / \mathrm{dI}$ is negative as the current rises up to about $500 \mathrm{~A}$, and is zero for higher currents up to $1500 \mathrm{~A}$. The axial position variation of the arc is therefore not able to move the regime where $\mathrm{dU} / \mathrm{dI}$ is negative towards higher currents. However, moving the arc further downstream helps reducing random voltage fluctuations, which might also be beneficial for passive oscillation breakers, since the voltage current characteristic is more predictable This stabilization seems to have more influence on the maximum arc voltage though, and reduces the steepness of the $\mathrm{dU} / \mathrm{dI}$ in the low current regime. It is difficult to say which of the presented setups is more favorable, since it is not clear if a stable but not so steep $\mathrm{dU} / \mathrm{dI}$ is more advantageous than a steep but strongly fluctuating dU/dI. So far, it is not clear what causes the voltage fluctuations, but at least part of it can be attributed to arc root 
movements. The experiments were recorded with a high speed camera, and when the downstream contact is close to the throat, the arc root often does not stay at the tip of the downstream electrode, but moves along the side of the pin for several centimeters, effectively doubling the length of the arc. One such occurrence is shown in figure 6. The downstream contact was upstream of the throat $(\beta<\gamma)$, so the arc was supposed to be in the converging nozzle section only. All measurements where this was the case showed significant voltage fluctuations whenever the arc root moved downstream along the contact, up to a voltage which was similar to measurements with the same $\alpha$, but a larger $\delta$ which were also taken, but not presented in the figures.

Ablation of the nozzle wall is also a factor, which requires more attention in the future. Weight measurements indicate a loss of PMMA wall material of more than 100 to 300 milligrams per shot, which is not negligible. Assuming this ablation only takes place during the measurement period when the current is high, a significant percentage of the arc power is needed to ablate this mass from the nozzle wall. It is suspected that the transition from negative $\mathrm{dU} / \mathrm{dI}$ to a constant voltage around $500 \mathrm{~A}$ is associated with the onset of ablation. Another factor is the resulting additional mass flow. Pressure measurements indicate that the mass flow of the blowing gas is reduced to almost zero while the current is above $1000 \mathrm{~A}$. This is only possible if the arc clogs most of the nozzle throat, and the remaining cross section is filled by the flow of ablated wall material.

\section{CONCLUSION}

Although the primary goal of understanding the physics behind the $\mathrm{dU} / \mathrm{dI}$ curve is far from complete, the presented

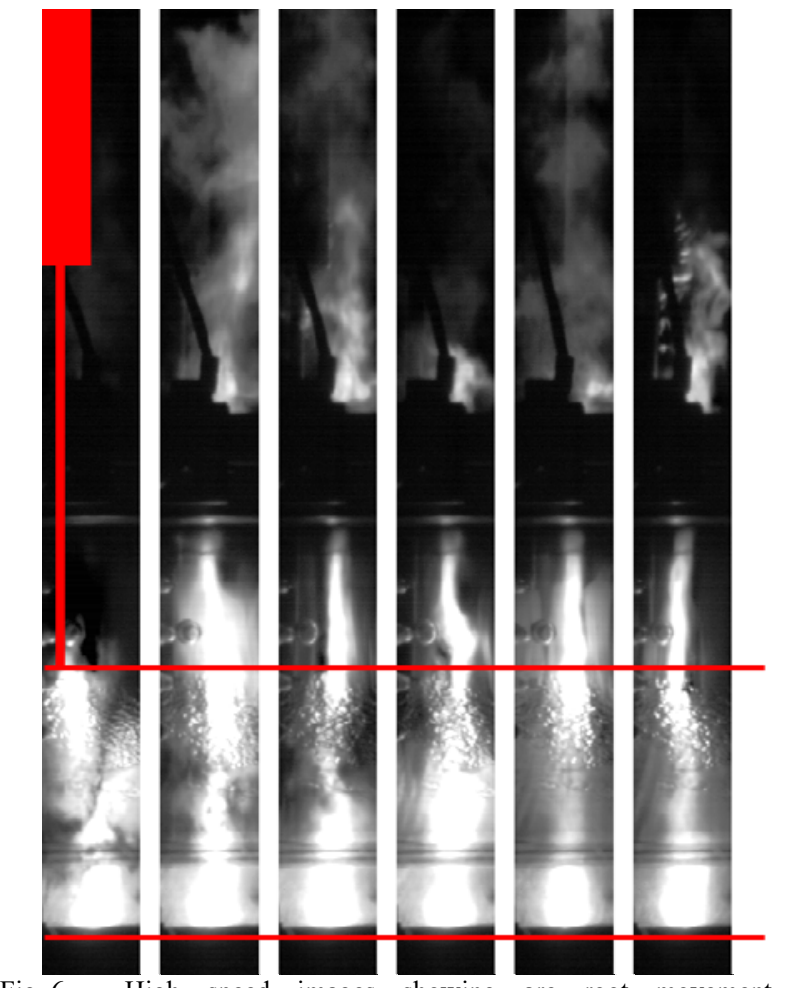

Fig. 6 High speed images showing arc root movement at downstream contact (indicated in red in leftmost frame). Both contact positions are indicated with vertical red lines. Exposure time is 2 us for each frame, time between frames is 200 us. The arc root moves downstream more than $80 \mathrm{~mm}$, i.e. the arc length more than doubles. method allows a comprehensive comparison of different geometries. So far little literature is available covering the fluctuations of the arc voltage away from a theoretical curve, but if those fluctuations dominate, they strongly influence the performance of passive resonance HVDC CB. So far, it seems that the electric field of the arc is largest at the nozzle throat, and the voltage drop per arc length is smaller in the converging and diverging parts of the nozzle. If the arc burns primarily in the converging part of the nozzle, the fluctuations are highest.

Future experimental work will include variations of the nozzle geometry, to analyze the influence of axial pressure gradients and more detailed analysis of nozzle ablation. This will be combined with theoretical studies, which builds on the simple arc models of [3]. The results of CFD simulations as in [12] and [10] also might be interesting, since they give access to parameters that are hard or impossible to measure.

\section{ACKNOWLEDGMENT}

The authors would like to thank the student Liu Mingkun who was a great help in the lab during experiments, and $\mathrm{ABB}$ Switzerland for financial support, as well as E. Panousis for the frequent discussions.

\section{REFERENCES}

M. M. Walter, "Switching Arcs in Passive Resonance HVDC

J. J. Lowke and H. C. Ludwig, "A simple model for high-current arcs stabilized by forced convection," J. Appl. Phys., vol. 46, no. 8, p. 3352, 1975.

D. T. Tuma and J. J. Lowke, "Prediction of properties of arcs stabilized by forced convection," J. Appl. Phys., vol. 46, no. 8, pp. $3361-3367,1975$.

W. Hermann, U. Kogelschatz, L. Niemeyer, K. Ragaller, and E. Schade, "Experimental and theoretical study of a stationary highcurrent arc in a supersonic nozzle flow," J. Phys. D. Appl. Phys., vol. 7, no. 12, pp. 1703-1722, Aug. 1974.

W. Hermann, U. Kogelschatz, K. Ragaller, and E. Schade, "Investigation of a cylindrical, axially blown, high-pressure arc," $J$. Phys. D. Appl. Phys., vol. 7, no. 4, pp. 607-619, Mar. 1974. E. . S. W . BÖTTICHER, U . KOGELSCHATZ, "Untersuchung quasistationärer Lichtbögen hoher Leistung bei starker axialer Gasströmung," 1972.

R. P. P. Smeets and V. Kertész, "Evaluation of high-voltage circuit breaker performance with a new validated arc model," IEE Proc. Gener. Transm. Distrib., vol. 147, no. 2, p. 121, Mar. 2000. A. Ahmethodžić, M. Kapetanović, K. Sokolija, R. P. P. Smeets, and V. Kertész, "Linking a physical arc model with a black box arc model and verification," IEEE Trans. Dielectr. Electr. Insul., vol. 18, no. 4, pp. 1029-1037, 2011.

H. Nordborg and A. A. Iordanidis, "Self-consistent radiation based modelling of electric arcs: I. Efficient radiation approximations," $J$. Phys. D. Appl. Phys., vol. 41, no. 13, p. 135205, Jul. 2008.

Y. Li, M. Rong, C. Niu, Y. Wu, M. Li, and F. Yang, "NUMERICAL STUDY ON SELF-EXCITED OSCILLATION SWITCHING CURRENT IN HVDC MRTB," in 20th International Conference on Gas Discharges and their Applications, 2014, pp. $183-186$.

A. Ritter, L. S. J. Bort, and C. M. Franck, "Five Years of Pulsed Current Testing for HVDC Switchgear," in IEEE International Conference on High Voltage Engineering and Application, 2016, p. (in press).

A. A. Iordanidis and C. M. Franck, "Self-consistent radiation-based simulation of electric arcs: II. Application to gas circuit breakers," $J$. Phys. D. Appl. Phys., vol. 41, no. 13, p. 135206, Jul. 2008. Circuit Breakers," 2013. 
This is the author's version of an article that has been published in the conference proceedings. Changes were made to this version prior to publication. The final version of record is available at https://doi.org/10.1109/ICHVE.2016.7800700 